\title{
Short-course Rifaximin therapy efficacy and lactulose hydrogen breath test in Chinese patients with diarrhea-predominant irritable bowel syndrome
}

\author{
Xiaojun Zhuang ${ }^{\dagger}$, Zhenyi Tian ${ }^{\dagger}$, Mei Luo and Lishou Xiong ${ }^{*}$
}

\begin{abstract}
Background: Gut microbiota alterations including small intestinal bacterial overgrowth (SIBO) might play a role in pathogenesis of irritable bowel syndrome (IBS). Rifaximin could effectively and safely improve IBS symptoms. The aim of this study was to investigate the effect of rifaximin on Gastrointestinal (GI) symptoms, quality of life (QOL) and SIBO eradication in Chinese IBS-D patients.

Methods: This study included 78 IBS-D patients defined by the Rome IV criteria. Patients received $400 \mathrm{mg}$ rifaximin twice daily for 2 weeks and 10-week follow-up. Gl symptoms were assessed at week 0, 2, 4, 8 and 12. QOL and lactulose hydrogen breath test (LHBT) results were estimated at week 0 and 4.

Results: All participants showed significant improvements in Gl symptom subdomains after rifaximin treatment (all $P<0.05)$, which could maintain at least 10 weeks of follow-up. Additionally, QOL scores were increased with concomitant improvement of clinical symptoms (all $P<0.05$ ). The 45 rifaximin-responsive patients $(57.7 \%)$ achieved significantly greater $\mathrm{Gl}$-symptom improvement than non-responders (all $P<0.05$ ). No Gl symptoms were associated with SIBO (all $P>0.05$ ). SIBO normalization after rifaximin treatment measured by LHBT was found in $44.4 \%$ (20/45) of patients with SIBO before treatment.

Conclusion: A short course (2 weeks) of rifaximin improved Gl symptoms and QOL in Chinese IBS-D patients whether they had SIBO or not. However, the efficacy of rifaximin could not be explained by the successful eradication of SIBO. Further studies on the therapeutic mechanisms of rifaximin in IBS are urgently needed.
\end{abstract}

Keywords: Irritable bowel syndrome, Rifaximin therapy, Small intestinal bacterial overgrowth, Quality of life

\section{Background}

Irritable bowel syndrome (IBS) is one of the most common functional bowel disorders, with a relapsing and remitting natural history characterized by abdominal pain that is associated with defecation or alterations in bowel habits [1]. The prevalence of IBS around the world is

\footnotetext{
* Correspondence: xionglishou@163.com

${ }^{\dagger}$ Xiaojun Zhuang and Zhenyi Tian contributed equally to this work. Department of Gastroenterology and Hepatology, the First Affiliated Hospital of Sun Yat-Sen University, Guangzhou 510080, China
}

approximately $7-21 \%$; it is $1-16 \%$ in China, but the prevalence differs depending on regions and diagnostic criteria [2, 3]. Dissatisfaction and comorbidities of traditional treatment are associated with a significant reduction in the quality of life (QOL) and growing social, sanitary and economic burden worldwide [4-6]. Patients are stratified into four subtypes based on the predominant bowel habit: constipation-predominant IBS (IBS-C), diarrhea-predominant IBS (IBS-D), mixed IBS (IBS-M) and unclassified IBS (IBS-U) [1]. Although the precise

(c) The Author(s). 2020 Open Access This article is licensed under a Creative Commons Attribution 4.0 International License, which permits use, sharing, adaptation, distribution and reproduction in any medium or format, as long as you give appropriate credit to the original author(s) and the source, provide a link to the Creative Commons licence, and indicate if changes were made. The images or other third party material in this article are included in the article's Creative Commons licence, unless indicated otherwise in a credit line to the material. If material is not included in the article's Creative Commons licence and your intended use is not permitted by statutory regulation or exceeds the permitted use, you will need to obtain permission directly from the copyright holder. To view a copy of this licence, visit http://creativecommons.org/licenses/by/4.0/ The Creative Commons Public Domain Dedication waiver (http://creativecommons.org/publicdomain/zero/1.0/) applies to the data made available in this article, unless otherwise stated in a credit line to the data. 
etiology of IBS remains unknown, the possible mechanisms include visceral hypersensitivity, gut motility dysfunction, immunomodulation disturbances, gut microbiota alterations and an imbalance in brain-gut axis interactions [7-10]. In addition, new-onset IBS symptoms following acute infectious gastroenteritis might also suggest a microbial pathogenesis for IBS [11].

Alterations in the quantity or composition of the gut microbiota with subsequent metabolic disturbances have been observed in patients with IBS. In a recent systematic review, increased abundances of Enterobacteriaceae and Lactobacillaceae at the family level and Bacteroides at the genus level were found in patients with IBS compared with controls, whereas the abundance of the order uncultured Clostridiales I, and the genera Faecalibacterium and Bifidobacterium were decreased in IBS patients [10]. Moreover, we previously reported alterations in the abundance of predominant fermenting bacteria involved in the pathophysiology of IBS-D (such as Bacteroidales and Clostridiales) [12]. Furthermore, an association between IBS and small intestinal bacterial overgrowth (SIBO) has been observed in some patients with IBS, although the causal relationship between SIBO and IBS remains to be elucidated [13-18]. SIBO might partly explain IBS symptoms, such as bloating, abdominal pain and changes in bowel habits. A definite diagnosis of SIBO is characterized by greater than $10^{5}$ microorganisms $/ \mathrm{ml}$ with poly-microbial flora in cultures of duodenal or jejunal fluid [19]. However, SIBO is diagnosed by various breath tests clinically, and the lactulose hydrogen breath testing (LHBT) is most commonly used, as intestinal samples are difficult to obtain [2022]. Gut microbiota alterations indicate that the manipulation of the composition of the intestinal microbiota with probiotics, prebiotics, antibiotics, dietary interventions and fecal microbiota transplantation may be useful treatment approaches [23].

Rifaximin, as a gastrointestinal (GI)-specific broadspectrum antibiotic, shows activity against both grampositive and gram-negative, anaerobic and aerobic bacteria [24]. Since it displays low systemic absorption and no clinically significant interactions with other drugs, rifaximin may be a promising treatment for IBS, mainly due to its ability to act on IBS pathogenesis by modulating gut microbiota, altering bacterial metabolism, preserving epithelial function and reducing proinflammatory cytokine production [25-27]. Additionally, prior studies on rifaximin in nonconstipated IBS patients with SIBO indicated that rifaximin treatment is effective in improving IBS symptoms and eradicating SIBO [28-32]. However, there are few studies on the association of GI symptoms and QOL with LHBT results in the Chinese population.

The overall aim of this study was to explore whether rifaximin treatment improves GI symptoms (abdominal discomfort, abdominal distension, abdominal pain, defecatory urgency, diarrhea and incomplete evacuation) and QOL (physical functioning, role-physical, bodily pain, general health, vitality, social functioning, roleemotional and mental health) in Chinese IBS patients. We hypothesized that rifaximin treatment could relieve GI symptoms and optimize QOL by normalizing SIBO a measured by the LHBT.

\section{Methods}

\section{Ethics statement}

This study was conducted at the Department of Gastroenterology and Hepatology, the First Affiliated Hospital of Sun Yat-sen University, from December 2016 to December 2018. The protocol was approved by the Medical Ethics Committee of the First Affiliated Hospital of Sun Yat-sen University, and all patients provided written informed consent. The ClinicalTrials.gov ID for the study is NCT02565654.

\section{Study subjects}

Seventy-eight patients with IBS-D were recruited into this study by two gastroenterologists with expertise in IBS. The inclusion criteria were men or women aged 18 years and above who met the Rome IV criteria for IBS-D, symptoms for more than 6 months, and patients with IBS symptoms as mentioned and normal appearance of the gastrointestinal mucosa. The exclusion criteria were clinical evidence of inflammatory bowel disease, a history of duodenal or gastric ulcers, diverticulitis or infectious gastroenteritis, abdominal surgery, cardiac, pulmonary, hepatic, renal or metabolic disease, use of antibiotics, probiotics, prebiotics, corticosteroids, proton-pump inhibitors, or IBS prescription medications within the last 4 weeks. A colonoscopy was performed on all patients to rule out organic disease.

\section{Study design and procedures}

All participants received $400 \mathrm{mg}$ rifaximin (Xifaxan', ALFASIGMA S.p.A., Bologna, Italy) twice daily for 2 weeks. Then, they were further followed-up for an additional 10 weeks after treatment cessation. For recruited patients, they were informed to not to take any other prebiotics, probiotics and antibiotic but rifaximin throughout the observation period. All investigators were asked to complete GI symptom questionnaire and an IBS-relevant QOL questionnaire based on the Medical Outcomes Study (MOS) item short-form health survey (SF-36). The symptoms were recorded in a diary at baseline, the end of the treatment (week 2), end of the 2-week follow-up (week 4), end of the 6-week follow-up (week 8), and the end of the 10-week follow-up (week 12). The assessed symptoms were abdominal discomfort, abdominal distension, abdominal pain, diarrhea, defecatory urgency and 
incomplete evacuation; the severity of GI symptoms was rated using a 7 -point Likert scale $(0=$ not at all, $1=$ hardly, $2=$ somewhat, $3=$ moderately, $4=$ a good deal, $5=$ a great deal, and $6=$ a very great deal). In addition, a QOL questionnaire was completed by IBS-D patients at baseline and at the end of the 2-week posttreatment period. The SF-36 is a 36-item questionnaire that measures 8 domains relevant to patients with IBS: (1) Physical Functioning, (2) Role-physical, (3) Bodily pain, (4) General Health, (5) Vitality, (6) Social Functioning, (7) Role-Emotional, and (8) Mental Health. Finally, all patients received an LHBT at baseline and the end of 2 weeks after rifaximin treatment.

\section{Evaluation of SIBO by LHBT}

The LHBT was performed according to a standard protocol. Patients did not receive any antibiotics, probiotics, prebiotics, or laxatives in the 4 weeks preceding the test. To minimize basal $\mathrm{H}_{2}$ excretion, IBS-D patients were asked to avoid foods containing complex carbohydrates (bread, potato, and corn) and fiber in the previous evening and fasted for at least $12 \mathrm{~h}$ before the breath test. Cigarette smoking and physical exercise were not allowed for $2 \mathrm{~h}$ before and during the test. On the day of testing, patients washed their mouths with $20 \mathrm{ml}$ of 0.05\% chlorhexidine (Koutai, Shenzhen, China) to eliminate the fermentation by oropharyngeal bacteria flora. LHBT was performed in IBS-D patients using a gas analyzer (GastroLyzer R Breath Hydrogen Monitor; Bedfont Science Ltd., UK). Immediately before the test, a sample of expired air was taken to assess the basal $\mathrm{H}_{2}$ concentration. Then, $10 \mathrm{~g}$ of lactulose dissolved in 100 $\mathrm{ml}$ of water was administered within $30 \mathrm{~s}$, and the expired air was sampled every 30 min over the next 3 consecutive hours by a trained study coordinator.

According to the literature and our previous results $[12,33,34]$, LHBT was considered indicative of the presence of SIBO when (i) a baseline value of $\mathrm{H}_{2} \geq 20 \mathrm{ppm}$ and/or (ii) a $>20 \mathrm{ppm}$ increase in $\mathrm{H}_{2}$ over basal values occurred within 90 min of lactulose administration.

\section{Outcome evaluation}

The primary endpoint was to assess the improvement in GI symptoms and QOL after 2 weeks of rifaximin treatment in the Chinese population. The secondary endpoint was to compare the LHBT results before and after treatment with rifaximin. We also explored the response rate to rifaximin treatment by analyzing the selfreported GI symptoms, and the response to treatment was defined as a more than $50 \%$ improvement in the global GI symptoms 2 weeks after the cessation of treatment. Finally, we sought to search for symptoms closely associated with SIBO.

\section{Statistical analysis}

All statistical analyses were performed using SPSS version 23.0 (SPSS, Inc., Chicago, IL, United States) and Graph Prism version 7.0 (GraphPad Software, Inc., La Jolla, CA, United States). Continuous data were analyzed using Student's t-test or Mann-Whitney U-test where appropriate. Categorical data were analyzed using a chisquare test. Pearson correlation coefficient analysis was used to assess the relationship between GI symptoms and SIBO. All tests for significance were two-sided and $P<0.05$ was considered statistically significant.

\section{Results}

Demographic and clinical characteristics of patients Seventy-eight patients (33.5 years [18-58], 52 [66.7\%] male) with IBS-D were enrolled in this study, and all participants completed a 12-week follow-up. Though IBS-D patients were more often male, the difference in age between the LHBT-positive and LHBT-negative groups was nonsignificant. Table 1 summarizes the demographic and clinical characteristics of all IBS-D patients. At baseline, 45 patients $(29 / 16)$ with SIBO were younger than those $(23 / 10)$ with a negative LHBT result $(32.13 \pm 7.48$ vs $37.24 \pm 9.95, P=0.016)$. In addition, there was no significant difference in the GI symptoms and QOL scores between the LHBT-negative and LHBT-positive groups. Moreover, no GI symptoms were found to be associated with the presence of SIBO (Table 2).

Table 1 Demographic and clinical characteristics of all included patients at baseline

\begin{tabular}{llll}
\hline Clinical factors & LHBT $(+)$ & LHBT $(-)$ & $P$ value \\
\hline Age (mean, years) & $32.13 \pm 7.48$ & $37.24 \pm 9.95$ & 0.016 \\
Gender (M/F) & $29 / 16$ & $23 / 10$ & 0.627 \\
Gl symptoms (mean) & $17.04 \pm 5.02$ & $17.42 \pm 4.40$ & 0.724 \\
Abdominal discomfort & $2.31 \pm 1.38$ & $2.55 \pm 1.23$ & 0.432 \\
Abdominal distension & $1.84 \pm 1.35$ & $2.00 \pm 1.17$ & 0.589 \\
Abdominal pain & $3.07 \pm 1.37$ & $2.45 \pm 1.37$ & 0.056 \\
Defecatory urgency & $3.78 \pm 1.31$ & $3.79 \pm 1.29$ & 0.973 \\
Diarrhea & $4.11 \pm 3.56$ & $3.64 \pm 1.06$ & 0.460 \\
Incomplete evacuation & $2.38 \pm 1.35$ & $3.00 \pm 1.39$ & 0.053 \\
Quality of life (mean) & $506.97 \pm 126.70$ & $477.82 \pm 105.95$ & 0.273 \\
Physical Functioning & $94.78 \pm 11.03$ & $93.03 \pm 6.49$ & 0.384 \\
Role-physical & $61.11 \pm 40.68$ & $52.27 \pm 40.68$ & 0.347 \\
Bodily pain & $57.72 \pm 20.48$ & $57.27 \pm 21.06$ & 0.449 \\
General Health & $43.33 \pm 19.86$ & $43.55 \pm 16.13$ & 0.958 \\
Vitality & $57.11 \pm 16.57$ & $55.91 \pm 19.86$ & 0.778 \\
Social Functioning & $75.67 \pm 20.94$ & $68.32 \pm 21.79$ & 0.138 \\
Role-Emotional & $55.55 \pm 42.05$ & $44.44 \pm 37.89$ & 0.226 \\
Mental Health & $61.69 \pm 16.79$ & $63.03 \pm 13.42$ & 0.696 \\
\hline
\end{tabular}




\section{Effect of rifaximin on SIBO}

For the subjects with SIBO before treatment, 25 (44.4\%) had a negative LHBT after 2 weeks of rifaximin treatment (week 4). Furthermore, patients who received rifaximin treatment more often tended to have a negative LHBT (45/33 [42.3\%] vs $25 / 53$ [67.9\%], $P=0.001)$ and had reduced hydrogen production. As shown in Table 3, there was significant difference in SIBO rate of Chinese IBS-D patients before and after rifaximin treatment (57.7, 95\% CI, $46.5-68.9 \%$ vs $32.1,95 \%$ CI: $21.5-42.6 \%$; $P<0.001$ ). In addition, there was no significant difference in age and gender between patients with and without LHBT normalization after 2 weeks of rifaximin therapy.

\section{Effect of rifaximin on $\mathrm{Gl}$ symptoms}

A symptomatic evaluation after 2 weeks of rifaximin treatment might show improvements before the LHBT normalizes (Fig. 1). The IBS symptoms of abdominal pain, abdominal discomfort, abdominal distension, diarrhea, defecatory urgency and incomplete evacuation improved significantly after rifaximin treatment, and the symptom relief persisted for at least 10 weeks during the follow-up period (all $P<0.05$ ). In addition, 45 (57.7\%) patients experienced a clinical response accompanied by a global IBS symptoms score reduction of at least 50\% $(5.36 \pm 3.27$ vs $13.79 \pm 5.21, P<0.001)$. The response group reported a full recovery or greater improvement in their symptoms than the nonresponse group, showing a significant difference in every GI symptom (Table 4). However, the difference in age and gender between the response and nonresponse groups was not statistically significant. For the IBS-D patients with SIBO, the GI symptoms showed significant improvement in each of the six symptom scores and in the global score after SIBO eradication through rifaximin treatment. Nevertheless, subjects without SIBO eradication exhibited a similar resolution in five GI symptoms, but not abdominal discomfort, suggesting that the effect of rifaximin in IBS-D is not explained by SIBO eradication. In contrast, LHBT-negative patients at baseline showed significant

Table 2 Correlation analysis between SIBO and GI symptoms

\begin{tabular}{lll}
\hline & \multicolumn{2}{l}{ SIBO } \\
\cline { 2 - 3 } & $\rho$ & $P$ value \\
\hline Abdominal discomfort & 0.081 & 0.483 \\
Abdominal distension & 0.083 & 0.468 \\
Abdominal pain & 0.231 & 0.052 \\
Defecatory urgency & 0.013 & 0.909 \\
Diarrhea & 0.064 & 0.578 \\
Incomplete evacuation & 0.199 & 0.081 \\
\hline
\end{tabular}

Note: $\rho$, Spearman rank correlation coefficient; SIBO, small intestinal bacterial overgrowth;

Gl symptoms, gastrointestinal symptoms
Table 3 SIBO rate in Chinese IBS-D patients pre- and postrifaximin treatment

\begin{tabular}{lclll}
\hline & LHBT & & $\begin{array}{l}95 \% \mathrm{Cl} \\
(\%)\end{array}$ & $\begin{array}{l}P \\
\text { nylue }\end{array}$ \\
\cline { 2 - 4 } & Positive/N (\%) & Negative/N (\%) & & \\
\hline Pre-treatment & $45(57.7)$ & $33(42.3)$ & $46.5-68.9$ & $<0.001$ \\
Post-treatment & $25(32.1)$ & $53(67.9)$ & $21.5-42.6$ & \\
\hline
\end{tabular}

Note: SIBO, Small intestinal bacterial overgrowth; IBS, Irritable bowel syndrome; IBS-D, diarrhea-predominant IBS

improvement in each of the six GI symptoms after treatment. Finally, IBS-D patients with SIBO or without SIBO at week 4 recorded similar GI symptoms scores, regardless of whether they succeeded in eradicating SIBO (Table 5).

\section{Effect of rifaximin on the QOL}

At baseline, all participants reported severely reduced QOL scores. Fortunately, total QOL scores significantly increased 2 weeks after the completion of treatment (week 4), indicating QOL improvement (Fig. 2). Compared to the nonresponse group, the response group reported significant alterations in five domains of QOL, with no significant difference in vitality, role-emotional and mental health (Table 4). Additionally, there was no significant difference in any of the eight domain scores or global QOL score between LHBT-positive and LHBT-negative groups. For the LHBT-positive IBS-D patients at baseline, bodily pain and general health improved significantly regardless of whether SIBO was successfully eliminated after rifaximin treatment. In contrast, a significant increase in seven QOL domain scores was observed in LHBT-negative patients at baseline with no significant difference in physical functioning after 2 weeks of rifaximin treatment. Finally, IBS-D patients with SIBO or without SIBO at week 4 recorded similar QOL scores, regardless of whether they succeeded in eradicating SIBO (Table 5).

\section{Adverse events}

No patient developed any adverse events during rifaximin administration, except for two patients who reported transient nausea during rifaximin treatment. Overall, the treatment was well tolerated.

\section{Discussion}

The findings of this study suggest that a short course (2 weeks) of rifaximin therapy is safe and efficacious for the treatment of IBS-D patients as assessed using the ROME IV criteria. The GI symptom relief, QOL improvement and SIBO normalization after rifaximin treatment observed in our study imply that rifaximin is an effective option for the treatment of IBS-D. Furthermore, the effectiveness of the short-course rifaximin treatment was sustained for at least 12 weeks after treatment. To our knowledge, this is the first study evaluating the effect of 

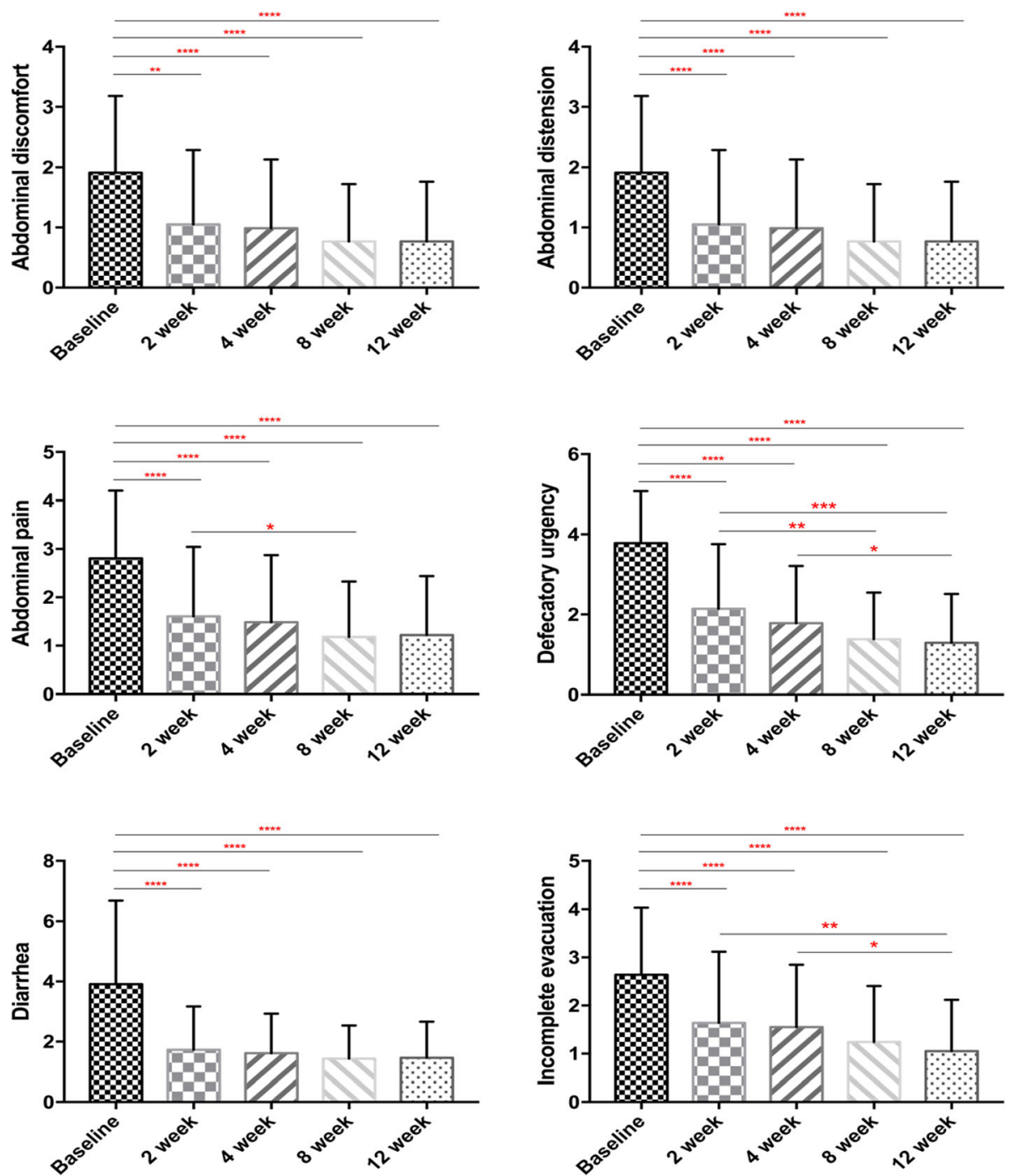

Fig. $1 \mathrm{Gl}$ symptoms scores in IBS-D patients at different times during the study. Note: ${ }^{*} P<0.05,{ }^{* *} P<0.01$, ${ }^{* * *} P<0.001$; IBS, irritable bowel syndrome; IBS-D, diarrhea-predominant IBS; GI symptoms, gastrointestinal symptoms

rifaximin on GI symptoms and QOL based on SIBO in Chinese patients with IBS-D.

As previously stated, the etiological and symptomatic manifestation of IBS and SIBO may overlap, and SIBO has been postulated to be a pathophysiological mechanism for IBS. Moreover, SIBO is in fact associated with IBS-like symptoms, such as bloating, abdominal pain, and a change in bowel habits. The frequency of SIBO among IBS patients ranges between 4 and $78 \%$, and the variations in prevalence of SIBO in previous studies might be attributable to differences in the geographical origins of the studied populations, different criteria for the diagnosis of IBS and methods for the diagnosis of SIBO using different breath tests [33-35]. The response to rifaximin treatment in IBS-D patients has been shown to correlate with the normalization of the LHBT results [32, 36, 37]. In our study, $57.7 \%$ of the included patients had a positive LHBT, and 20 showed LHBT normalization after 2 weeks of rifaximin treatment, with a SIBO eradication rate of $44.4 \%$. In addition, the LHBT-positive subjects were younger than the LHBT-negative subjects. In contrast to our study, a recent meta-analysis involving 32 studies reported that the overall eradication rate according to an intention-to-treat analysis was 70.8\% (95\% CI: $\left.61.4-78.2 ; \mathrm{I}^{2}=89.4 \%\right)$ and according to a per-protocol analysis was $72.9 \%$ (95\% CI: 65.5-79.8; $\mathrm{I}^{2}=87.5 \%$ ) [32]. However, another metaanalysis of eight studies showed that the overall breathtest normalization rate with rifaximin was $49.5 \%$, which is somewhat similar to the result of our study [38]. The marked discrepancy in rates of SIBO eradication might be related to geographical, dietary or ethnicity differences in the microbiomes of the study populations or the dose of 
Table 4 Comparison between the Response and Non-Response Groups after rifaximin treatment at week 4

\begin{tabular}{llll}
\hline Clinical factors & $\begin{array}{l}\text { Response group } \\
(n=45)\end{array}$ & $\begin{array}{l}\text { Non-response group } \\
(n=33)\end{array}$ & $P$ value \\
\hline Age (mean, years) & $34.82 \pm 9.04$ & $33.58 \pm 8.84$ & 0.544 \\
Gender (M/F) & $31 / 14$ & $21 / 12$ & 0.627 \\
LHBT (+/-) & $18 / 27$ & $8 / 25$ & 0.145 \\
Gl symptoms (mean) & $5.36 \pm 3.27$ & $13.79 \pm 5.21$ & $<0.001$ \\
Abdominal discomfort & $0.87 \pm 0.89$ & $2.45 \pm 1.23$ & $<0.001$ \\
Abdominal distension & $0.49 \pm 0.70$ & $1.67 \pm 1.29$ & $<0.001$ \\
Abdominal pain & $0.91 \pm 0.93$ & $2.27 \pm 1.53$ & $<0.001$ \\
Defecatory urgency & $1.09 \pm 1.13$ & $2.73 \pm 1.26$ & $<0.001$ \\
Diarrhea & $1.02 \pm 0.97$ & $2.42 \pm 1.23$ & $<0.001$ \\
Incomplete evacuation & $0.98 \pm 1.03$ & $2.33 \pm 1.22$ & $<0.001$ \\
Quality of life (mean) & $616.34 \pm 84.06$ & $545.34 \pm 94.70$ & 0.001 \\
Physical Functioning & $97.33 \pm 4.47$ & $94.85 \pm 5.93$ & 0.048 \\
Role-physical & $82.78 \pm 24.90$ & $67.42 \pm 36.70$ & 0.031 \\
Bodily pain & $77.34 \pm 13.70$ & $62.36 \pm 19.88$ & $<0.001$ \\
General Health & $59.22 \pm 16.52$ & $45.64 \pm 16.70$ & 0.001 \\
Vitality & $67.33 \pm 15.06$ & $61.67 \pm 14.23$ & 0.094 \\
Social Functioning & $85.63 \pm 10.52$ & $76.67 \pm 19.98$ & 0.012 \\
Role-Emotional & $76.30 \pm 32.28$ & $72.73 \pm 30.57$ & 0.621 \\
Mental Health & $70.40 \pm 16.30$ & $64.00 \pm 18.08$ & 0.112 \\
\hline
\end{tabular}

Table 5 Gl symptoms and QOL comparisons between IBS-D patients with and without SIBO eradication after rifaximin treatment

\begin{tabular}{llll}
\hline Clinical factors & LHBT $(+)$ & LHBT $(-)$ & $P$ value \\
\hline Gl symptoms (mean) & $7.88 \pm 6.15$ & $8.95 \pm 5.23$ & 0.531 \\
Abdominal discomfort & $1.64 \pm 1.29$ & $1.20 \pm 1.20$ & 0.243 \\
Abdominal distension & $0.92 \pm 1.04$ & $0.90 \pm 1.29$ & 0.956 \\
Abdominal pain & $1.64 \pm 1.35$ & $1.45 \pm 1.28$ & 0.631 \\
Defecatory urgency & $1.32 \pm 1.44$ & $2.05 \pm 1.28$ & 0.078 \\
Diarrhea & $1.20 \pm 1.29$ & $1.95 \pm 1.10$ & 0.051 \\
Incomplete evacuation & $1.16 \pm 1.11$ & $1.55 \pm 1.15$ & 0.256 \\
Quality of life (mean) & $579.93 \pm 106.11$ & $599.05 \pm 88.29$ & 0.513 \\
Physical Functioning & $96.20 \pm 4.63$ & $98.25 \pm 3.73$ & 0.107 \\
Role-physical & $70.00 \pm 35.36$ & $81.25 \pm 25.49$ & 0.222 \\
Bodily pain & $71.92 \pm 14.29$ & $73.68 \pm 15.41$ & 0.697 \\
General Health & $53.60 \pm 19.72$ & $53.85 \pm 16.69$ & 0.963 \\
Vitality & $63.80 \pm 14.74$ & $64.00 \pm 15.01$ & 0.965 \\
Social Functioning & $84.57 \pm 12.92$ & $82.95 \pm 13.41$ & 0.685 \\
Role-Emotional & $72.00 \pm 32.89$ & $81.67 \pm 33.29$ & 0.336 \\
Mental Health & $67.84 \pm 17.42$ & $63.40 \pm 18.55$ & 0.417 \\
\hline
\end{tabular}

Note: IBS, irritable bowel syndrome; IBS-D, diarrhea-predominant IBS; GI symptoms, gastrointestinal symptoms; QOL, quality of life; SIBO, small intestinal bacterial overgrowth rifaximin. The findings of our study suggest that either SIBO plays a limited role in causing IBS-associated gut microbiota disturbances or that LHBT is not a good test to measure SIBO.

There was no significant difference in GI symptoms and QOL scores between the LHBT-positive and LHBTnegative groups. After 2 weeks of rifaximin treatment, all individual and global symptoms displayed instant improvement, and these effects lasted for at least 10 weeks during the follow-up period. However, not all patients showed a desirable response to rifaximin therapy according to the formal prespecified criteria for a response. In the LHBT-positive group, the response rate was relatively high $(40.0 \%)$ compared with that in the LHBTnegative group (24.2\%), but the difference was not statistically significant. More importantly, participants with LHBT normalization after treatment appeared to experience symptomatic improvement in all of the six symptoms, whereas those without SIBO eradication showed similar symptom relief, with the exception of abdominal discomfort. However, more severe diarrhea was recorded in subjects with LHBT normalization than those without LHBT normalization, which means that this nonabsorbable antimicrobial agent did not completely reverse the gut microflora dysbiosis when eradicating SIBO. In addition, subjects with a negative LHBT at baseline also achieved individual and global GI symptom improvements that persisted after rifaximin intervention. In the well-known TARGET 1 and TARGET II studies, only $40 \%$ of patients responded to rifaximin, but treatment with rifaximin for 2 weeks provided significant relief of IBS symptoms including bloating, abdominal pain, and loose or watery stools [28]. The inconsistent response to rifaximin in various studies may be due to IBS heterogeneity, and LHBT normalization might not be a good marker to assess the response to rifaximin. In contrast to our study, an open-label study from Europe reported an improvement in individual symptoms (abdominal pain, diarrhea, and bloating) as well global symptoms with $800 \mathrm{mg} /$ day rifaximin for 2 weeks [39]. Recently, a study of retreatment with rifaximin showed a $33 \%$ response rate in the rifaximin group compared to $25 \%$ in the placebo group $(P=0.02)$, consistent with FDA guidelines for the clinical assessment of IBS drugs in the TARGET 3 study [40].

At baseline, all participants reported reduced QOL scores. Interestingly, the IBS-QOL overall and all subdomain scores improved from baseline for up to 2 weeks posttreatment and were accompanied by symptom relief in the included patients. Indeed, responders had a significantly greater improvement in the overall QOL score than nonresponders, which implies that a sufficient improvement in patient clinical symptoms guarantees that their QOL improves. Furthermore, rifaximin treatment 

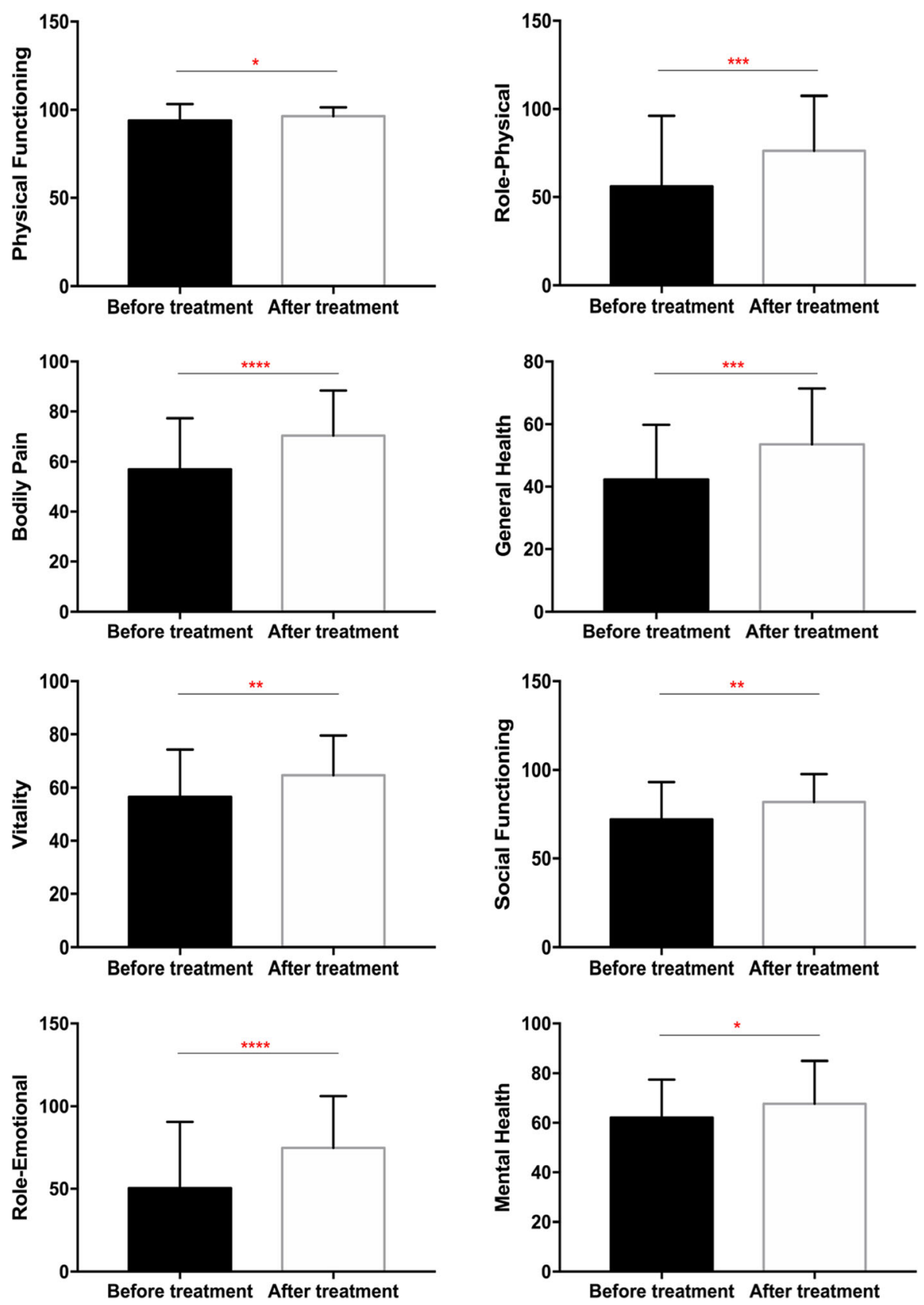

Fig. 2 QOL scores comparison in IBS-D patients pre- and post-rifaximin treatment. Note: ${ }^{*} P<0.05$, ${ }^{* *} P<0.01$, ${ }^{* *} P<0.001$; IBS, irritable bowel syndrome; IBS-D, diarrhea-predominant IBS; QOL, Quality of life

significantly impacted bodily pain and general health in patients with a positive LHBT, regardless of whether SIBO was successfully eradicated. Interestingly, our findings indicate that treatment with rifaximin favorably improves the total QOL and seven subdomain scores in LHBTnegative patients with IBS-D, which is consistent with previously reported data [41]. Similar effects have been seen in another study, the findings of which suggested that the increased improvement in QOL following repeat treatment with rifaximin is associated with a reduced chance of subsequent symptom relapse [42]. However, rifaximin was not effective in improving IBS symptoms and QOL in Gulf War veterans with non-constipated IBS [43]. Finally, LHBT-positive and LHBT-negative IBS-D patients did not differ significantly in their reported post rifaximin total QOL or subscale scores. 
Rifaximin was approved by the US Food and Drug Administration in 2015 to treat adults with IBS-D [44]. Although the mechanism of action of rifaximin in IBS is complex, a leading hypothesis proposes that rifaximin modulates intestinal flora imbalances. Mounting evidence has shown that rifaximin treatment induces alterations in the abundance of specific bacterial populations rather than affecting the overall composition of the microbiota in the treated subjects and has no apparent detrimental effects on gut microbiota [45-47]. On the one hand, rifaximin shows a potent killing effect on common SIBO pathogens $[48,49]$. On the other hand, rifaximin appears to increase the abundance of certain potentially beneficial bacteria, such as Faecalibacterium prausnitzii, but reduces the abundance of detrimental bacteria such as Clostridium. In addition to the direct antibiotic effects of rifaximin on gut microbiota, rifaximin impacts the function of the gut microbiota (i.e., metabolism, adherence and virulence) [50-52]. Alterations in certain lipid species, saturated and unsaturated fatty acids, and products of carbohydrate metabolism were found in several studies focused on rifaximin treatment for IBS; these alterations might have beneficial effects on various symptoms (improved barrier function of the small bowel and reduced visceral hyperalgesia) of GIrelated disease. For example, a study from Bajaj et al. found that alteration of gut bacterial linkages with metabolites rather than significant change in microbial abundance after rifaximin therapy, which especially linked to ammonia, aromatic amino acids and oxidative stress [50]. Furthermore, rifaximin could inhibit bacterial interactions with the host to reduce detrimental bacterial colonization, infection and the activation of the host immune response to prevent mucosal inflammation by reducing the level of proinflammatory mediators [53]. In addition, rifaximin is able to reduce bacterial virulence and translocation, has anti-inflammatory properties by increasing the relative abundance of Faecalibacterium prausnitzi endowed with powerful anti-inflammatory activities [54]. Taken together, these results show that the beneficial effects and safety of rifaximin treatment might be partly accounted for by resetting the gut microenvironment and modulating the inflammatory environment.

There are several limitations in this study. It was conducted in a single center with a relatively small sample size and open-label design so that conclusions should be drawn cautiously. Further limitations of our study are no control group and the lack of randomization. Additionally, the validity and interpretation of the LHBT for the diagnosis of SIBO is an ongoing controversy. The greatest weakness of the study is that the potential mechanisms by which rifaximin beneficially affects IBS-D patients with definite SIBO were not elaborated comprehensively. Nevertheless, it might be noteworthy that this is the first study to show that short-course rifaximin therapy is an appropriate treatment option for Chinese IBS-D patients.

\section{Conclusion}

In conclusion, a short course of rifaximin treatment significantly improved the GI symptoms and QOL of Chinese IBS-D patients in this study, and 2-week rifaximin treatment led to the sustained improvement of IBS symptoms for at least 10 weeks, which is consistent with multiple previous large clinical trials of single and repeat treatment cycles. However, the efficacy of rifaximin could not be explained by the successful eradication of SIBO. More therapeutic mechanisms of rifaximin for IBS-D patients are warranted in further studies.

\section{Abbreviations}

IBS: Irritable bowel syndrome; IBS-D: patients with diarrhea-predominant IBS; SIBO: Small intestinal bacterial overgrowth; LHBT: Lactulose hydrogen breath test; QOL: Quality of life

\section{Acknowledgments}

None.

\section{Authors' contributions}

LX was responsible for the study concept and design, critical revision of the manuscript for important intellectual content. XZ was responsible for the study concept and design, acquisition of data, analysis and interpretation of data and drafting of the manuscript. ZT and ML assisted in collecting data and conducting the statistical analysis. All authors have read and approved this version of the article. Neither the entire paper nor any part of its content has been published or has been accepted elsewhere.

\section{Funding}

This study was funded by the National Natural Science Foundation of China (81970471) and Alfasigma Pharmaceutical Company. The funders had a role in study design, decision to publish and preparation of the manuscript. No additional external funding was received for this study.

Availability of data and materials

All data and materials are not available in this study, and are available from the corresponding author on reasonable requests.

\section{Ethics approval and consent to participate}

This study was approved by the Medical Ethical Committee of the First Affiliated Hospital of Sun Yat-sen University. All patients had signed informed consent.

\section{Consent for publication}

Not applicable.

\section{Competing interests}

Authors declare that they have no competing interests.

Received: 13 March 2020 Accepted: 5 June 2020

Published online: 12 June 2020

\section{References}

1. Drossman DA, Hasler WL. Rome IV-functional Gldisorders: disorders of gutbrain interaction. Gastroenterology. 2016;150(6):1257-61.

2. Chey WD, Kurlander J, Eswaran S. Irritable bowel syndrome: a clinical review. JAMA. 2015;313(9):949-58.

3. Xiong LS, Chen MH, Chen HX, Xu AG, Wang WA, Hu PJ, et al. A populationbased epidemiologic study of irritable bowel syndrome in South China: stratified randomized study by cluster sampling. Aliment Pharmacol Ther. 2004;19(11):1217-24. 
4. Buono JL, Carson RT, Flores NM. Health-related quality of life, work productivity, and indirect costs among patients with irritable bowel syndrome with diarrhea. Health Qual Life Outcomes. 2017;15(1):35.

5. Agarwal N, Spiegel BM. The effect of irritable bowel syndrome on healthrelated quality of life and health care expenditures. Gastroenterol Clin N Am. 2011:40(1):11-9.

6. Gwee KA, Ghoshal UC, Chen M. Irritable bowel syndrome in Asia: pathogenesis, natural history, epidemiology, and management. J Gastroenterol Hepatol. 2018;33(1):99-110.

7. Holtmann GJ, Ford AC, Talley NJ. Pathophysiology of irritable bowel syndrome. Lancet Gastroenterol Hepatol. 2016;1(2):133-46.

8. Ford AC, Lacy BE, Talley NJ. Irritable bowel syndrome. N Engl J Med. 2017; 376(26):2566-78

9. Moser G, Fournier C, Peter J. Intestinal microbiome-gut-brain axis and irritable bowel syndrome. Wien Med Wochenschr. 2018;168(3-4):62-6.

10. Pittayanon R, Lau JT, Yuan Y, Leontiadis Gl, Tse F, Surette M, et al. Gut microbiota in patients with irritable bowel syndrome-a systematic review. Gastroenterology. 2019;157(1):97-108.

11. Klem F, Wadhwa A, Prokop LJ, Sundt WJ, Farrugia G, Camilleri M, et al. Prevalence, risk factors, and outcomes of irritable bowel syndrome after infectious enteritis: a systematic review and meta-analysis. Gastroenterology. 2017; 152(5):1042-54.

12. Zhuang $X$, Tian Z, Li L, Zeng Z, Chen M, Xiong L. Fecal microbiota alterations associated with diarrhea-predominant irritable bowel syndrome. Front Microbiol. 2018;9:1600.

13. Pimentel $\mathrm{M}$, Chow EJ, Lin HC. Eradication of small intestinal bacterial overgrowth reduces symptoms of irritable bowel syndrome. Am J Gastroenterol. 2000;95(12):3503-6.

14. Ghoshal UC, Srivastava D. Irritable bowel syndrome and small intestinal bacterial overgrowth: meaningful association or unnecessary hype. World Gastroenterol. 2014;20(10):2482-91.

15. Ghoshal UC, Shukla R, Ghoshal U. Small intestinal bacterial overgrowth and irritable bowel syndrome: a bridge between functional organic dichotomy. Gut Liver. 2017;11(2):196-208.

16. Lin HC. Small intestinal bacterial overgrowth: a framework for understanding irritable bowel syndrome. JAMA. 2004;292(7):852-8.

17. Shah ED, Basseri RJ, Chong K, Pimentel M. Abnormal breath testing in IBS: a meta-analysis. Dig Dis Sci. 2010;55(9):2441-9.

18. Chen B, Kim JJ, Zhang Y, Du L, Dai N. Prevalence and predictors of small intestinal bacterial overgrowth in irritable bowel syndrome: a systematic review and meta-analysis. J Gastroenterol. 2018;53(7):807-18.

19. Grace E, Shaw C, Whelan K, Andreyev HJ. Review article: small intestinal bacterial overgrowth-prevalence, clinical features, current and developing diagnostic tests, and treatment. Aliment Pharmacol Ther. 2013;38(7):674-88.

20. Kerlin P, Wong L. Breath hydrogen testing in bacterial overgrowth of the small intestine. Gastroenterology. 1988;95(4):982-8.

21. Nucera G, Gabrielli M, Lupascu A, Lauritano EC, Santoliquido A, Cremonini F, et al. Abnormal breath tests to lactose, fructose and sorbitol in irritable bowel syndrome may be explained by small intestinal bacterial overgrowth. Aliment Pharmacol Ther. 2005;21(11):1391-5.

22. Ghoshal UC, Srivastava D, Ghoshal U, Misra A. Breath tests in thediagnosis of small intestinal bacterial overgrowth in patients with irritable bowel syndrome in comparison with quantitative upper gut aspirate culture. Eur J Gastroenterol Hepatol. 2014;26(7):753-60.

23. Rodino-Janeiro BK, Vicario M, Alonso-Cotoner C, Pascua-Garcia R, Santos J. A review of microbiota and irritable bowel syndrome: future in therapies. Adv Ther. 2018;35(3):289-310.

24. Scarpignato C, Pelosini I. Rifaximin, a poorly absorbed antibiotic: pharmacology and clinical potential. Chemotherapy. 2005;51(Suppl 1):36-66.

25. Pimentel M. Review article: potential mechanisms of action of rifaximin in the management of irritable bowel syndrome with diarrhoea. Aliment Pharmacol Ther. 2016:43(Suppl 1):37-49.

26. Acosta A, Camilleri M, Shin A, Linker Nord S, O'Neill J, Gray AV, et al. Effects of Rifaximin on transit, permeability, fecal microbiome, and organic acid excretion in irritable bowel syndrome. Clin Transl Gastroenterol. 2016;7:e173.

27. Kane JS, Ford AC. Rifaximin for the treatment of diarrhea-predominant irritable bowel syndrome. Expert Rev Gastroenterol Hepatol. 2016;10(4): $431-42$.

28. Pimentel M, Lembo A, Chey WD, Zakko S, Ringel $Y, Y u$ J, et al. Rifaximin therapy for patients with irritable bowel syndrome without constipation. N Engl J Med. 2011;364(1):22-32.
29. Sharara Al, Smalley W, Heidelbaugh JJ, Mounzer R, Sidani S, Elhaji I. A randomized double-blind placebo-controlled trial of rifaximin in patients with abdominal bloating and flatulence. Am J Gastroenterol. 2006;101(2): 326-33.

30. Lembo A, Pimentel M, Rao SS, Schoenfeld P, Cash B, Weinstock LB, et al. Repeat treatment with Rifaximin is safe and effective in patients with diarrhea-predominant irritable bowel syndrome. Gastroenterology. 2016; 151(6):1113-21.

31. Menees SB, Maneerattannaporn M, Kim HM, Chey WD. The efficacy and safety of rifaximin for the irritable bowel syndrome: a systematic review and meta-analysis. Am J Gastroenterol. 2012;107(1):28-35.

32. Gatta L, Scarpignato C. Systematic review with meta-analysis: rifaximin is effective and safe for the treatment of small intestine bacterial overgrowth. Aliment Pharmacol Ther. 2017;45(5):604-16.

33. Gasbarrini A, Corazza GR, Gasbarrini G, Montalto M, Di Stefano M, Basilisco $\mathrm{G}$, et al. Methodology and indications of H2-breath testing in gastrointestinal diseases: the Rome consensus conference. Aliment Pharmacol Ther. 2009;29(Suppl 1):1-49.

34. Rezaie A, Buresi M, Lembo A, Lin H, McCallum R, Rao S, et al. Hydrogen and methane-based breath testing in gastrointestinal disorders: the north American consensus. Am J Gastroenterol. 2017:112(5):775-84.

35. Ford AC, Spiegel BM, Talley NJ, Moayyedi P. Small intestinal bacterial overgrowth in irritable bowel syndrome: systematic review and metaanalysis. Clin Gastroenterol Hepatol. 2009;7(12):1279-86.

36. Bae S, Lee KJ, Kim YS, Kim KN. Determination of rifaximin treatment period according to lactulose breath test values in nonconstipated irritable bowel syndrome subjects. J Korean Med Sci. 2015;30(6):757-62.

37. Pimentel $M$, Chow EJ, Lin HC. Normalization of lactulose breath testing correlates with symptom improvement in irritable bowel syndrome: a double-blind, randomized, placebo-controlled study. Am J Gastroenterol. 2003;98(2):412-9.

38. Shah SC, Day LW, Somsouk M, Sewell JL. Meta-analysis: antibiotic therapy for small intestinal bacterial overgrowth. Aliment Pharmacol Ther. 2013; 38(8):925-34.

39. Sharara Al, Aoun E, Abdul-Baki H, Mounzer R, Sidani S, Elhajj I. A randomized double-blind placebo-controlled trial of rifaximin in patients with abdomina bloating and flatulence. Am J Gastroenterol. 2006;101(2):326-33.

40. Weinberg DS, Smalley W, Heidelbaugh JJ, Sultan S. American Gastroenterological Association Institute guideline on the pharmacologica management of irritable bowel syndrome. Gastroenterology. 2014;147(5): 1146-8.

41. Sherwin LB, Deroche CB, Krisanabud P, Matteson-Kome M, Bechtold M, Ruppar T. Adherence to short-course pharmacotherapy in adults with irritable bowel syndrome. West J Nurs Res. 2019;1918313053.

42. Cash BD, Pimentel M, Rao S, Weinstock L, Chang L, Heimanson Z, et al. Repeat treatment with rifaximin improves irritable bowel syndrome-related quality of life: a secondary analysis of a randomized, double-blind, placebocontrolled trial. Ther Adv Gastroenterol. 2017;10(9):689-99.

43. Tuteja AK, Talley NJ, Stoddard GJ, Verne GN. Double-blind placebocontrolled study of Rifaximin and lactulose hydrogen breath test in gulf war veterans with irritable bowel syndrome. Dig Dis Sci. 2019;64(3):838-45.

44. Xifaxan (rifaximin) tablets, for oral use (package insert). Salix Pharmaceuticals: Bridgewater, NJ 2015.

45. Soldi S, Vasileiadis S, Uggeri F, Campanale M, Morelli L, Fogli MV, et al. Modulation of the gut microbiota composition by rifaximin in nonconstipated irritable bowel syndrome patients: a molecular approach. Clin Exp Gastroenterol. 2015;8:309-25.

46. Fodor AA, Pimentel M, Chey WD, Lembo A, Golden PL, Israel RJ, et al. Rifaximin is associated with modest, transient decreases in multiple taxa in the gut microbiota of patients with diarrhoea-predominant irritable bowel syndrome. Gut Microbes. 2019;10(1):22-33.

47. Chang C. Short-course therapy for diarrhea-predominant irritable bowel syndrome: understanding the mechanism, impact on gut microbiota, and safety and tolerability of rifaximin. Clin Exp Gastroenterol. 2018;11: 335-45.

48. Jiang ZD, Ke S, Palazzini E, Riopel L, DuPont HL. In vitro activity and fecal concentration of rifaximin after oral administration. Antimicrob Agents Chemother. 2000:44(8):2205-6.

49. Pistiki A, Galani I, Pyleris E, Barbatzas C, Pimentel M, Giamarellos-Bourboulis EJ. In vitro activity of rifaximin against isolates from patients with small intestinal bacterial overgrowth. Int J Antimicrob Agents. 2014;43(3):236-41. 
50. Bajaj JS, Heuman DM, Sanyal AJ, Hylemon PB, Sterling RK, Stravitz RT, et al. Modulation of the metabiome by rifaximin in patients with cirrhosis and minimal hepatic encephalopathy. PLoS One. 2013;8(4):e60042.

51. Kajander K, Myllyluoma E, Kyronpalo S, Rasmussen M, Sipponen P, Mattila I, et al. Elevated pro-inflammatory and lipotoxic mucosal lipids characterise irritable bowel syndrome. World J Gastroenterol. 2009;15(48):6068-74.

52. Jiang ZD, Ke S, DuPont HL. Rifaximin-induced alteration of virulence of diarrhoea-producing Escherichia coli and Shigella sonnei. Int J Antimicrob Agents. 2010;35(3):278-81.

53. Xu D, Gao J, Gillilland M, Wu X, Song I, Kao JY, et al. Rifaximin alters intestinal bacteria and prevents stress-induced gut inflammation and visceral hyperalgesia in rats. Gastroenterology. 2014;146(2):484-96.

54. Ponziani FR, Zocco MA, D'Aversa F, Pompili M, Gasbarrini A. Eubiotic properties of rifaximin: disruption of the traditional concepts in gut microbiota modulation. World J Gastroenterol. 2017;23(25):4491-9.

\section{Publisher's Note}

Springer Nature remains neutral with regard to jurisdictional claims in published maps and institutional affiliations.

Ready to submit your research? Choose BMC and benefit from:

- fast, convenient online submission

- thorough peer review by experienced researchers in your field

- rapid publication on acceptance

- support for research data, including large and complex data types

- gold Open Access which fosters wider collaboration and increased citations

- maximum visibility for your research: over $100 \mathrm{M}$ website views per year

At $\mathrm{BMC}$, research is always in progress.

Learn more biomedcentral.com/submissions 